\title{
Quantum Work Relations under trial Hamiltonians
}

\author{
Arun K. Pati ${ }^{(1) *}$, Mamata Sahoo ${ }^{(1,2)}$ and Biswajit $\operatorname{Pradhan}^{(1)}$ \\ (1) Institute of Physics, Bhubaneswar-751005, Orissa, India and \\ (2) Max Planck Institute for Colloids and Interfaces, \\ Science Park Golm, D-14424 Potsdam, Germany
}

(Dated: November 20, 2018)

\begin{abstract}
The universal quantum work relation connects a functional of an arbitrary observable averaged over the forward process to the free energy difference and another functional averaged over the time-reversed process. Here, we ask the question if the system is driven out of equilibrium by a different Hamiltonian rather than the original one during the forward process and similarly during the reversed process then how accurate is the quantum work relation. We present an inequality that must be satisfied when the system is driven out by such a trial Hamiltonian. This also answers the issue of accuracy of the Jarzynski relation with a trial Hamiltonian. We have shown that the correction term can be expressed as the averages of the difference operator between the accurate and trial Hamiltonians. This leads to a generalized version of the Bogoliubov inequality for the free energy differences.
\end{abstract}

\footnotetext{
*Email: akpati@iopb.res.in
} 


\section{INTRODUCTION}

In recent years work relations in nonequilibrium setting have attracted much attention. Jarzynski's equality [1] is an important step in that direction, where this provides a relation between the distribution of work performed on a classical system subjected to an external force and the free energy difference between the initial and final configurations. In particular, a finite system is prepared in a state of equilibrium with an environment at temperature $\mathrm{T}$. The system is driven with an external parameter from some initial value to a final value. During this process the Jarzynski equality connects the equilibrium information about the free energy to that of the nonequilibrium processes. The nonequilibrium work relation has been proved from a generalized version of the fluctuation theorem [2]. The Jarzynski equality has been generalized to quantum domain also [3 -5, 9]. Kurchan has considered extension of this relation in a quantum setting where he has used measurement based schemes for the system of interest at the initial and final time and related the work performed to the energy difference between the final and initial eigenstates [3]. Monnai [4] has derived the quantum version of the Jarzynski equality in terms of the microscopic reversibility and has explored the equilibrium information about the free energy of a system when it is thermally isolated and is driven externally out of equilibrium. The derivation of the work relation by Monnai [4] and Tasaki [5] is based on the twice measurements scheme. For more details regarding the twice measurements scheme, readers are advised to see [6, 7]. In addition, there is quantum work operator approach to derive the work relation which can give the required energy difference when the system is driven out of equilibrium [8]. The quantum analog of the Jarzynski equality has also been derived for closed [3, 5, 10] as well as open quantum systems [1]].

Very recently, Andrieux and Gaspard [12] have proved a universal quantum work relation which connects the average of a functional of an arbitrary observable during the forward process to that of another functional averaged over the time-reversed process and the free energy difference. As shown in that paper many well known results follow from this universal work relation. For example, the Jarzynski equality [1], quantum Green-Kubo relations [13], and Casimir-Onsager [14] relations can be obtained from the universal work relation.

In statistical mechanics approximate methods are very useful when dealing with complex systems that consist of many particles. For instance computing the free energy and the partition function of statistical systems are in general intractable. Only for few special models one may be able to calculate such thermodynamic quantities. In these situations one replaces the original Hamiltonian (which may be difficult to handle) by a trial Hamiltonian (or an approximate Hamiltonian). Under such approximations, proving various thermodynamic relations are important and one must try to establish how accurate are those relations. In this paper, we ask the question if the system is driven out of equilibrium by a different Hamiltonian $H^{\prime}(t)$ rather than the original one during the forward process and similarly during the reversed process then how accurate is the universal quantum work relation. For complex systems testing the Jarzynski equality or the Andrieux-Gaspard relation may be equally difficult. If we imagine that $H^{\prime}(t)$ is a trial Hamiltonian then the inequality that we are going to prove in the sequel will tell us how accurate is the universal work relation or the Jarzynski relation as compared to the actual one. In general, $H^{\prime}(t)$ may contain different interaction or several external control parameters. In these cases this inequality will be of use in estimating the work performed on the system and the free energy differences. In particular, we have shown that the correction term can be explicitly expressed as the 
averages of the difference operator between the accurate and trial Hamiltonians. Therefore, the error in the free energy difference caused by the modelling procedure can be estimated by calculating the difference operator. This in turn leads to a generalized version of the Bogoliubov inequality for the free energy differences.

The organization of our paper is as follows. In section II, we introduce the universal work relation. In section III, we prove the main inequality for the universal work relation under the trial Hamiltonian. In section IV, we address the issue of accuracy of the Jarzynski relation under the trial Hamiltonian. Also, we prove a generalized version of the Bogoliubov inequality for the free energy differences. In section $\mathrm{V}$, we present yet another useful inequality for the Jarzynski relation in terms of the operator norm. Finally, we conclude with some discussions in section VI.

\section{UNIVERSAL WORK RELATION}

Before presenting the main result let us briefly introduce the setting in which the universal work relation of Andrieux and Gaspard [12] was proved. Let us imagine a system whose Hamiltonian is given by a Hermitian operator $H(t, \mathbf{R})$, where $\mathbf{R}$ can be some external parameters that change sign under the time-reversal (such as the magnetic field). Under the time reversal operator $\Theta$ the Hamiltonian transforms as $\Theta H(t, \mathbf{R}) \Theta=H(t,-\mathbf{R})$, with $\Theta$ being an anti-linear operator and $\Theta^{2}=I$. Initially the system is prepared in thermal equilibrium with the inverse temperature $\beta=\frac{1}{k_{B} \mathrm{~T}}$, where $k_{B}$ being the Boltzmann constant and $\mathrm{T}$ is the temperature. During the forward process, the system is driven out of equilibrium by a time dependent Hamiltonian $H(t, R)$. The initial state of the system is a canonical density matrix

$$
\rho(0)=\frac{e^{-\beta H(0, \mathbf{R})}}{Z(0)},
$$

where $Z(0)=\operatorname{Tr}\left[e^{-\beta H(0, \mathbf{R})}\right]$ is the partition function, with $\rho(0)^{\dagger}=\rho(0), \rho(0)>0$ and $\operatorname{Tr} \rho(0)=1$. The free energy $F(0)$ of the system at the initial time is given via the partition function $Z(0)=e^{-\beta F(0)}$. We allow the system to evolve from the initial time to some final time $t=T$. The forward evolution is governed by the evolution equation

$$
i \hbar \frac{d U_{F}(t, \mathbf{R})}{d t}=H(t, \mathbf{R}) U_{F}(t, \mathbf{R}) .
$$

If we consider the Heisenberg representation then the observables evolve as $A_{F}(t)=$ $U_{F}(t)^{\dagger} A U_{F}(t)$ and similarly for the time-dependent Hamiltonian we have $H_{F}(t)=$ $U_{F}(t)^{\dagger} H(t, \mathbf{R}) U_{F}(t)$. During the backward process the external parameters are reversed. The system is driven by a time-reversed Hamiltonian $H(T-t,-\mathbf{R})$ with a canonical density matrix

$$
\rho(T)=\frac{e^{-\beta H(T,-\mathbf{R})}}{Z(T)} .
$$

Note that the time dependence in the Hamiltonian is rescaled so that the initial time during the reverse process corresponds to $t=0$. The partition function and the free energy for this

process are given by the relation $Z(T)=\operatorname{Tr}\left[e^{-\beta H(T,-\mathbf{R})}\right]=e^{-\beta F(T)}$. The system is allowed 
to evolve until $t=T$ where the Hamiltonian at the end of the process is $H(0,-\mathbf{R})$. The evolution equation during the backward process is given by

$$
i \hbar \frac{d U_{R}(t, \mathbf{R})}{d t}=H(T-t, \mathbf{R}) U_{R}(t, \mathbf{R}) .
$$

Now it can be shown that the forward and the backward evolution operators are related via

$$
\Theta U_{F}(T-t, \mathbf{R}) U_{F}^{\dagger}(t, \mathbf{R}) \Theta=U_{R}(t,-\mathbf{R}) .
$$

In the Heisenberg picture the observables which have definite parity under time reversal obey the following relation during the forward and the backward process

$$
A_{F}(t)= \pm U_{F}^{\dagger}(T) \Theta A_{R}(T-t) \Theta U_{F}(T),
$$

where $A_{R}(T-t)=U_{R}^{\dagger}(T-t) A U_{R}(T-t)$. Now, the universal work relation of Andrieux and Gaspard states the following.

Theorem: For an arbitrary time-independent observable $A$ with a definite parity the functional relation

$$
\left\langle e^{\int_{0}^{T} d t \lambda(t) A_{F}(t)} e^{-\beta H_{F}(T)} e^{\beta H(0)}\right\rangle_{\rho(0), F}=e^{-\beta \Delta F}\left\langle e^{ \pm \int_{0}^{T} d t \lambda(T-t) A_{R}(t)}\right\rangle_{\rho(T), R}
$$

connects the averages during the forward and the backward processes. Here, $\lambda(t)$ is an arbitrary function, $\Delta F=F(T)-F(0)$ is the free energy difference of the two equilibrium states. The average of an operator $O$ during the forward and the backward processes are defined via $\left\langle O>_{\rho(0), F}=\operatorname{Tr}[\rho(0) O]\right.$ and $<O>_{\rho(T), R}=\operatorname{Tr}[\rho(T) O]$, respectively. As mentioned, the Jarzynski relation can be obtained from (7). This relation also provides the linear response theory of an arbitrary observable.

\section{INEQUALITY FOR QUANTUM WORK RELATION}

In this section, we prove the main inequality for the universal work relation under the trial Hamiltonian. Suppose that the system is not driven by the original Hamiltonian but by a different Hamiltonian $H^{\prime}\left(t, \mathbf{R}^{\prime}\right)$ (we allow the new Hamiltonian to depend on a different set of external parameters $\mathbf{R}^{\prime}$ ). This can be a trial Hamiltonian or an approximate Hamiltonian or it could consist of perturbation to the original Hamiltonian. The question that we are concerned here is how stable or how accurate is the universal work relation with such a trial Hamiltonian that is different than the original one. To carry out the stability analysis of quantum work relation we use adiabatic representation theory where the original Hamiltonian $H(t, \mathbf{R})$ and the trial Hamiltonian $H^{\prime}\left(t, \mathbf{R}^{\prime}\right)$ are assumed to have set of instantaneous eigenstates at the initial and the final time. Otherwise the results presented here are quite general.

Imagine that the system is driven by a trial Hamiltonian $H^{\prime}\left(t, \mathbf{R}^{\prime}\right)$. The system starts from a canonical density matrix

$$
\rho^{\prime}(0)=\frac{e^{-\beta H^{\prime}\left(0, \mathbf{R}^{\prime}\right)}}{Z^{\prime}(0)}
$$

at time $t=0$ and evolves as before until time $t=T$. During the forward process the observables evolve according to the trial Hamiltonian as $A_{F}^{\prime}(t)=U_{F}^{\prime}(t)^{\dagger} A U_{F}^{\prime}(t)$ and similarly 
for the time-dependent Hamiltonian we have $H_{F}^{\prime}(t)=U_{F}^{\prime}(t)^{\dagger} H^{\prime}\left(t, \mathbf{R}^{\prime}\right) U_{F}^{\prime}(t)$. The backward process follows according to the trial Hamiltonian starting from a canonical density matrix

$$
\rho^{\prime}(T)=\frac{e^{-\beta H^{\prime}\left(T,-\mathbf{R}^{\prime}\right)}}{Z^{\prime}(T)} .
$$

Here, the primed quantities have similar meaning when the system is governed by the trial Hamiltonian. The partition function is given by $Z^{\prime}(T)=\operatorname{Tr}\left[e^{-\beta H^{\prime}\left(T,-\mathbf{R}^{\prime}\right)}\right]$. Now, with this trial Hamiltonian one can show that the universal work relation reads as

$$
\left\langle e^{\int_{0}^{T} d t \lambda(t) A_{F}^{\prime}(t)} e^{-\beta H_{F}^{\prime}(T)} e^{\beta H^{\prime}(0)}\right\rangle_{\rho^{\prime}(0), F}=e^{-\beta \Delta F^{\prime}}\left\langle e^{ \pm \int_{0}^{T} d t \lambda(T-t) A_{R}^{\prime}(t)}\right\rangle_{\rho^{\prime}(T), R}
$$

This connects the functional averages of an arbitrary observable during the forward and the backward process when the system is driven by a trial Hamiltonian. Here, $\lambda(t)$ being an arbitrary function, $\Delta F^{\prime}=F^{\prime}(T)-F^{\prime}(0)$ being the free energy difference of the two equilibrium states. The averages during forward and the backward processes are defined as $\langle O\rangle_{\rho^{\prime}(0), F}=\operatorname{Tr}\left[\rho^{\prime}(0) O\right]$ and $\langle O\rangle_{\rho^{\prime}(T), R}=\operatorname{Tr}\left[\rho^{\prime}(T) O\right]$, respectively.

What we prove is that the following inequality is satisfied

$$
\left\langle e^{\int_{0}^{T} d t \lambda(t) A_{F}^{\prime}(t)} e^{-\beta H_{F}^{\prime}(T)} e^{\beta H^{\prime}(0)}\right\rangle_{\rho^{\prime}(0), F} \leq e^{-\beta \Delta F} e^{\beta\left(\langle V(0)\rangle_{\rho(0), F}-\langle V(T)\rangle_{\rho^{\prime}(T), R}\right)}\left\langle e^{ \pm \int_{0}^{T} d t \lambda(T-t) A_{R}^{\prime}(t)}\right\rangle_{\rho^{\prime}(T), R},
$$

where $V(t)=H^{\prime}\left(t, \mathbf{R}^{\prime}\right)-H(t, \mathbf{R})$ is the difference between the trial and the actual Hamiltonian. The above inequality can also be stated as

$$
\frac{\left\langle e^{\int_{0}^{T} d t \lambda(t) A_{F}^{\prime}(t)} e^{-\beta H_{F}^{\prime}(T)} e^{\beta H^{\prime}(0)}\right\rangle_{\rho^{\prime}(0), F}}{\left\langle e^{ \pm \int_{0}^{T} d t \lambda(T-t) A_{R}^{\prime}(t)}\right\rangle_{\rho^{\prime}(T), R}} \leq \frac{\left\langle e^{\int_{0}^{T} d t \lambda(t) A_{F}(t)} e^{-\beta H_{F}(T)} e^{\beta H(0)}\right\rangle_{\rho(0), F}}{\left\langle e^{ \pm \int_{0}^{T} d t \lambda(T-t) A_{R}(t)}\right\rangle_{\rho(T), R}} e^{\beta\left(\langle V(0)\rangle_{\rho(0), F}-\langle V(T)\rangle_{\rho^{\prime}(T), R}\right)} .
$$

To prove the above inequality we assume that the time-dependent Hamiltonians $H(t)$ and $H^{\prime}(t)$ have instantaneous eigenstates at time $t=0$ and $t=T$. Let the original Hamiltonian satisfies an eigenvalue equation at time $t=0$ as $H(0, \mathbf{R})\left|\psi_{n}(0)\right\rangle=E_{n}(0, \mathbf{R})\left|\psi_{n}(0)\right\rangle$. The partition function at $t=0$ can be written as $Z(0)=\operatorname{Tr}\left[e^{-\beta H(0, \mathbf{R})}\right]=\sum_{n}\left\langle\psi_{n}(0)\left|e^{-\beta H(0, \mathbf{R})}\right| \psi_{n}(0)\right\rangle$. During the forward process consider the diagonal elements of the canonical density matrices $\rho(0)$ and $\rho^{\prime}(0)$ in the eigenbasis $\left|\psi_{n}(0)\right\rangle$. Thus, we have two probability distributions $p_{n}(0)$ and $p_{n}^{\prime}(0)$ as given by

$$
\begin{aligned}
& p_{n}(0)=\frac{e^{-\beta E_{n}(0, \mathbf{R})}}{Z(0)} \\
& p_{n}^{\prime}(0)=\left\langle\psi_{n}(0)\left|\frac{e^{-\beta H^{\prime}\left(0, \mathbf{R}^{\prime}\right)}}{Z^{\prime}(0)}\right| \psi_{n}(0)\right\rangle .
\end{aligned}
$$

For these two probability distributions $p_{n}(0)$ and $p_{n}^{\prime}(0)$ the relative entropy of $p$ with respect to $p^{\prime}$ (also called the Kullback-Leibler distance) is defined by

$$
R\left(p, p^{\prime}\right)=\sum_{n} p_{n}(0) \log \frac{p_{n}(0)}{p_{n}^{\prime}(0)} \geq 0
$$


It is a convex function of $p_{n}(0)$ and is always non-negative and equals zero only if $p_{n}(0)=$ $p_{n}^{\prime}(0)$. Using this, we can write the inequality as

$$
\sum_{n} p_{n}(0) \log p_{n}(0) \geq \sum_{n} p_{n}(0) \log p_{n}^{\prime}(0)
$$

Now, using the expressions for $p_{n}(0)$ and $p_{n}^{\prime}(0)$, we can write the above inequality as

$$
\sum_{n} p_{n}(0)\left[-\beta E_{n}(0, \mathbf{R})-\log Z(0)\right] \geq \sum_{n} p_{n}(0)\left\langle\psi_{n}(0)\left|\left(-\beta H^{\prime}\left(0, \mathbf{R}^{\prime}\right)-\log Z^{\prime}(0)\right)\right| \psi_{n}(0)\right\rangle .(15
$$

In the above equation, on the right hand side we have used the Jensen inequality, i.e., $\left\langle e^{O}\right\rangle \geq e^{\langle O\rangle}$ which gives us

$$
\begin{aligned}
\log p_{n}^{\prime}(0) & =\log \left[\left\langle\psi_{n}(0)\left|\left(-\beta H^{\prime}\left(0, \mathbf{R}^{\prime}\right)-\log Z^{\prime}(0)\right)\right| \psi_{n}(0)\right\rangle\right] \\
& \geq\left\langle\psi_{n}(0)\left|\left(-\beta H^{\prime}\left(0, \mathbf{R}^{\prime}\right)-\log Z^{\prime}(0)\right)\right| \psi_{n}(0)\right\rangle
\end{aligned}
$$

Therefore, we have the following inequality that gives a lower bound on the partition function with the trial Hamiltonian at the initial time of forward process

$$
Z^{\prime}(0) \geq Z(0) e^{-\beta \operatorname{Tr}\left[\rho(0)\left(H^{\prime}\left(0, \mathbf{R}^{\prime}\right)-H(0, \mathbf{R})\right]\right.} .
$$

Next we derive an upper bound on the partition function with the trial Hamiltonian at the initial time of the backward process. During the backward process with a trial Hamiltonian the system starts from the density matrix as given in (9). Let us assume that the trial Hamiltonian satisfies an instantaneous eigenvalue equation at time $t=T$, i.e., $H^{\prime}\left(T,-\mathbf{R}^{\prime}\right)\left|\psi_{n}^{\prime}(T)\right\rangle=E_{n}^{\prime}\left(T,-\mathbf{R}^{\prime}\right)\left|\psi_{n}^{\prime}(T)\right\rangle$, where $E_{n}^{\prime}\left(T,-\mathbf{R}^{\prime}\right)$ is the eigenvalue. Now consider the diagonal elements of the canonical density matrices corresponding to the Hamiltonian $H(T,-\mathbf{R})$ and $H^{\prime}\left(T,-\mathbf{R}^{\prime}\right)$ in the eigenbasis of $\left|\psi_{n}^{\prime}(T)\right\rangle$. These probability distributions are given by

$$
\begin{aligned}
& p_{n}(T)=\left\langle\psi_{n}^{\prime}(T)\left|\frac{e^{-\beta H(T,-\mathbf{R})}}{Z(T)}\right| \psi_{n}^{\prime}(T)\right\rangle \\
& p_{n}^{\prime}(T)=\left\langle\psi_{n}^{\prime}(T)\left|\frac{e^{-\beta H^{\prime}\left(T,-\mathbf{R}^{\prime}\right)}}{Z^{\prime}(T)}\right| \psi_{n}^{\prime}(T)\right\rangle=\frac{e^{-\beta E_{n}^{\prime}\left(T,-\mathbf{R}^{\prime}\right)}}{Z^{\prime}(T)} .
\end{aligned}
$$

Let us consider the relative entropy of $p_{n}^{\prime}(T)$ with respect to $p_{n}(T)$ which is given by

$$
\sum_{n} p_{n}^{\prime}(T) \log \frac{p_{n}^{\prime}(T)}{p_{n}(T)} \geq 0 .
$$

Again using the non-negative property of the relative entropy we can write the above inequality as

$$
\sum_{n} p_{n}^{\prime}(T) \log p_{n}^{\prime}(T) \geq \sum_{n} p_{n}^{\prime}(T) \log p_{n}(T)
$$

Using the expressions for $p_{n}^{\prime}(T), p_{n}(T)$ and the Jensen inequality as before, we have

$$
\begin{array}{r}
\sum_{n} p_{n}^{\prime}(T)\left[-\beta E_{n}^{\prime}\left(T,-\mathbf{R}^{\prime}\right)-\log Z^{\prime}(T)\right] \geq \\
\sum_{n} p_{n}^{\prime}(T)\left\langle\psi_{n}^{\prime}(T)|(-\beta H(T,-\mathbf{R})-\log Z(T))| \psi_{n}^{\prime}(T)\right\rangle .
\end{array}
$$


This leads to an upper bound for the partition function with the trial Hamiltonian during the time-reversed process as given by

$$
Z^{\prime}(T) \leq Z(T) e^{-\beta \operatorname{Tr}\left[\rho^{\prime}(T)\left(H^{\prime}\left(T,-\mathbf{R}^{\prime}\right)-H(T,-\mathbf{R})\right)\right]} .
$$

From these two inequalities (17) and (22) we have

$$
\frac{Z^{\prime}(T)}{Z^{\prime}(0)} \leq \frac{Z(T)}{Z(0)} e^{\beta\left[\langle V(0)\rangle_{\rho(0), F}-\langle V(T)\rangle_{\rho^{\prime}(T), R}\right]}
$$

where $\langle V(0)\rangle_{\rho(0), F}=\operatorname{Tr}\left[\rho(0)\left(H^{\prime}\left(0, \mathbf{R}^{\prime}\right)-H(0, \mathbf{R}) \quad\right)\right]$ and $\langle V(T)\rangle_{\rho^{\prime}(T), R}=$ $\operatorname{Tr}\left[\rho^{\prime}(T)\left(H^{\prime}\left(T,-\mathbf{R}^{\prime}\right)-H(T,-\mathbf{R})\right)\right]$. By noting the fact that $\frac{Z^{\prime}(T)}{Z^{\prime}(0)}=e^{-\beta \Delta F^{\prime}}$ and $\frac{Z(T)}{Z(0)}=e^{-\beta \Delta F}$, we have the main inequality for the universal quantum work relation with a trial Hamiltonian as given in Eq.(11). Hence, the proof.

\section{JARZYNSKI RELATION WITH TRIAL HAMILTONIAN}

In this section, we address the question of the accuracy of the Jarzynski relation when the system is driven away from equilibrium by a trial Hamiltonian. Interestingly, the quantum version of the Jarzynski equality follows from the universal work relation of Andrieux and Gaspard [12]. If we set $\lambda=0$ in Eq.(7), we obtain

$$
\left\langle e^{-\beta H_{F}(T)} e^{\beta H(0)}\right\rangle_{F}=\left\langle e^{-\beta W}\right\rangle=e^{-\beta \Delta F}
$$

where the left hand side represents the average of the exponential of work performed on the system during the forward process and $\Delta F$ is the equilibrium free energy difference $F(T)-F(0)$. Now if one asks the question what happens to the Jarzynksi equality if we drive the system with a different Hamiltonian $H^{\prime}(t)$ rather than $H(t)$. Here, $H^{\prime}(t)$ could be a trial Hamiltonian, or could be some perturbation. Under such a situation how stable is the Jarzynski relation or the nonequilibrium work relation. Our inequality can answer this question. It is clear that if we drive the system with a different Hamiltonian $H^{\prime}\left(t, \mathbf{R}^{\prime}\right)$ then we will have the Jarzynski equality

$$
\left\langle e^{-\beta H_{F}^{\prime}(T)} e^{\beta H^{\prime}(0)}\right\rangle_{F}=\left\langle e^{-\beta W^{\prime}}\right\rangle=e^{-\beta \Delta F^{\prime}} .
$$

From our inequality (11), if we set $\lambda=0$, we will obtain the following inequality for the Jarzynski relation

$$
\begin{array}{r}
\left\langle e^{-\beta H_{F}^{\prime}(T)} e^{\beta H^{\prime}(0)}\right\rangle_{F}=e^{-\beta \Delta F^{\prime}} \leq e^{-\beta \Delta F} e^{\beta\left[\langle V(0)\rangle_{\rho(0), F}-\langle V(T)\rangle_{\rho^{\prime}(T), R}\right]} \\
\text { or } \quad\left\langle e^{-\beta W^{\prime}}\right\rangle \leq\left\langle e^{-\beta W}\right\rangle e^{\beta\left[\langle V(0)\rangle_{\rho(0), F}-\langle V(T)\rangle_{\rho^{\prime}(T), R}\right]}
\end{array}
$$

This tells us that the work performed by a trial Hamiltonian in going from the initial configuration to the final configuration must respect this bound. Eq.(26) is important in its own right. This shows that the correction term can be explicitly expressed as the averages of the difference operator between the accurate and trial Hamiltonians. By calculating the quantity $\left[\langle V(0)\rangle_{\rho(0), F}-\langle V(T)\rangle_{\rho^{\prime}(T), R}\right]$ the error in the free energy difference caused by the modelling procedure can be estimated directly. 
Furthermore, using the inequality (26), we can prove a generalized version of the Bogoliubov inequality. One version of the usual Bogoliubov inequality [15] can be stated as follows: Let $H$ be the original Hamiltonian and let $H^{\prime}$ be the trial Hamiltonian with the condition that $\operatorname{tr}\left(\rho^{\prime} H^{\prime}\right)=\operatorname{tr}\left(\rho^{\prime} H\right)$, i.e., the averages of both the Hamiltonians in the canonical state $\rho^{\prime}$ are same, then $F \leq F^{\prime}$. Here, $F$ is the free energy of the original Hamiltonian and $F^{\prime}$ is the free energy of the trial Hamiltonian. The Bogoliubov inequality has important application in the mean field theory. For example, by a variational method if we can minimize the free energy of the trial Hamiltonian we can get a better approximation to the exact free energy.

To prove the generalized version of the Bogoliubov inequality, we note from (26) that

$$
\Delta F^{\prime}-\Delta F \geq\left[\langle V(T)\rangle_{\rho^{\prime}(T), R}-\langle V(0)\rangle_{\rho(0), F}\right] .
$$

Now, if we impose the condition that $H(t)$ and $H^{\prime}(t)$ have same averages in the original and trial canonical state at time $t=0$ and $t=T$, then $\langle V(T)\rangle_{\rho^{\prime}(T), R}=0$ and $\langle V(0)\rangle_{\rho(0), F}=0$. Then it follows that

$$
\Delta F \leq \Delta F^{\prime}
$$

This can be regarded as time-dependent generalization of the Bogoliubov inequality which bounds the change in the free energy of original Hamiltonian with respect to perturbed one. This is an another important result. This inequality not only holds close to equilibrium but also hold in those situations which are far-from-equilibrium regime.

\section{INEQUALITY WITH OPERATOR NORM}

In this section, we prove an inequality for the Jarzynski relation which involves the norm of the operator $V(t)$. This may be useful when we do not have to calculate the averages of the operator $V(0)$ and $V(T)$ during the forward and backward processes, corresponding to the actual and trial Hamiltonians.

Consider the partition function for the trial Hamiltonian during the initial time of the reverse process. We have

$$
Z^{\prime}(T)=\operatorname{Tr}\left[e^{-\beta H^{\prime}\left(T,-\mathbf{R}^{\prime}\right)}\right] \leq \operatorname{Tr}\left[e^{-\beta H(T,-\mathbf{R})} e^{-\beta V(T)}\right],
$$

where we have used the inequality $\operatorname{Tr}\left[e^{A+B}\right] \leq \operatorname{Tr}\left[e^{A} e^{B}\right]$ for all self-adjoint operators.

On expressing the trace using the eigenbasis of $H(T,-\mathbf{R})$ we have

$$
\begin{aligned}
Z^{\prime}(T) & \leq \sum_{n}\left\langle\psi_{n}(T)\left|e^{-\beta H(T,-\mathbf{R})} e^{-\beta V(T)}\right| \psi_{n}(T)\right\rangle \\
& =\sum_{n}\left\langle\psi_{n}(T)\left|e^{-\beta H(T,-\mathbf{R})}\right| \psi_{n}(T)\right\rangle\left\langle\psi_{n}(T)\left|e^{-\beta V(T)}\right| \psi_{n}(T)\right\rangle .
\end{aligned}
$$

Now, we use the inequality $\left\|e^{A}\right\| \leq e^{\|A\|}$ which holds for all operators. The operator norm of a linear operator $A$ is defined as $\|A\|=\sup _{(\|\psi\|=1)}\|A(\psi)\|$. (Note that in the matrix notation, $A(\psi)=A|\psi\rangle$ and $\|A\|$ is equal to the square root of the largest eigenvalue of the symmetric matrix $A^{T} A$.)

Using this we have

$$
\begin{aligned}
Z^{\prime}(T) & \leq \sum_{n}\left\langle\psi_{n}(T)\left|e^{-\beta H(T,-\mathbf{R})}\right| \psi_{n}(T)\right\rangle e^{\beta\|V(T)\|} \\
& =Z(T) e^{\beta\|V(T)\|}
\end{aligned}
$$


This gives an upper bound for the partition function for the trial Hamiltonian at the initial time of the reverse process.

Now, let us consider the partition function $Z(0)$ with the Hamiltonian $H(0)$ during the initial time of the forward process. We have

$$
Z(0)=\operatorname{Tr}\left[e^{-\beta H(0, \mathbf{R})}\right] \leq \operatorname{Tr}\left[e^{-\beta H^{\prime}\left(0, \mathbf{R}^{\prime}\right)} e^{\beta V(0)}\right],
$$

where we have again used the inequality $\operatorname{Tr}\left[e^{A+B}\right] \leq \operatorname{Tr}\left[e^{A} e^{B}\right]$ which holds for all self-adjoint operators. We express the trace using the eigenbasis of $H^{\prime}(0, \mathbf{R})$. This gives us

$$
\begin{aligned}
Z(0) & \leq \sum_{n}\left\langle\psi_{n}^{\prime}(0)\left|e^{-\beta H^{\prime}\left(0, \mathbf{R}^{\prime}\right)} e^{\beta V(0)}\right| \psi_{n}^{\prime}(0)\right\rangle \\
& =\sum_{n}\left\langle\psi_{n}^{\prime}(0)\left|e^{-\beta H^{\prime}\left(0, \mathbf{R}^{\prime}\right)}\right| \psi_{n}^{\prime}(0)\right\rangle\left\langle\psi_{n}^{\prime}(0)\left|e^{\beta V(0)}\right| \psi_{n}^{\prime}(0)\right\rangle .
\end{aligned}
$$

Now, we use the inequality $\left\|e^{A}\right\| \leq e^{\|A\|}$ which holds for all operators. Using this we have

$$
\begin{aligned}
Z(0) & \leq \sum_{n}\left\langle\psi_{n}^{\prime}(0)\left|e^{-\beta H^{\prime}\left(0, \mathbf{R}^{\prime}\right)}\right| \psi_{n}^{\prime}(0)\right\rangle e^{\beta\|V(0)\|} \\
& =Z^{\prime}(0) e^{\beta\|V(0)\|} .
\end{aligned}
$$

This gives a lower bound for the partition function with the trial Hamiltonian during the initial time of the forward process.

On multiplying two inequalities (31) and (34), we have the new inequality for the Jarzynski relation under the trial Hamiltonian as given by

$$
\begin{aligned}
\left\langle e^{-\beta W^{\prime}}\right\rangle & =e^{-\beta \Delta F^{\prime}} \leq e^{-\beta \Delta F} e^{\beta\|V(0)\|} e^{\beta\|V(T)\|} \\
\text { or } \quad\left\langle e^{-\beta W^{\prime}}\right\rangle & \leq\left\langle e^{-\beta W}\right\rangle e^{\beta\|V(0)\|} e^{\beta\|V(T)\|} .
\end{aligned}
$$

This inequality may be often useful where we do not have to calculate explicitly the averages of the operator $V$ for the actual and trial states. Eq. (35) provides the exact estimation of the free energy difference between the initial and final states when the actual Hamiltonian is not the same as the trial or approximate Hamiltonian. With the help of the free energy, all the quantum equilibrium properties like different phases of a system and their physical properties like the phase transitions etc. can be exactly calculated. This relation is expressed in terms of an inequality which involves a correction term when the actual Hamiltonian is not same as the trial or approximate Hamiltonian. The correction term is expressed in the form of the norm of the operator $V$ at the initial and final time. In some physical situations it may be difficult to calculate exactly the averages of $V(T)$ or $V(0)$. Also, the free energy of a quantum system relative to an arbitrary reference state is often difficult to determine. In such situations, Eq.(35) may provide a practical tool to extract the quantum equilibrium information even if the averages of $V$ is explicitly not known. The norm of $V$ at the initial and final time is enough to give the required estimate.

\section{CONCLUSIONS}

Testing various thermodynamic relations for complex systems are not always amenable. One has to model the actual Hamiltonian of the system by a trial Hamiltonian and establish 
how accurate are those relations. In this paper, we have investigated the stability of the universal quantum work relation of Andrieux and Gaspard under a trial Hamiltonian and proved an inequality that must be respected if the system is driven away from equilibrium by a trial Hamiltonian rather than the actual one. In turn, our inequality also tells us how accurate is the famous Jarzynski relation under such trial Hamiltonians. We have shown, in particular, that the correction term can be explicitly expressed by the averages of the difference operator $V$ between the accurate and the trial Hamiltonians. Therefore, the free energy difference caused by the modelling procedure can be estimated by calculating the difference operator. We have also obtained a generalized version of the Bogoliubov inequality for the time-dependent trial Hamiltonians. This tells us that the change in the free energy corresponding to the actual Hamiltonian is always less than or equal to the change in the free energy in the trial case. Further, we have given an inequality using the operator norm where the correction term can be expressed in the form of the norm of the operator $V$ at the initial and final time. In future, we plan to apply this inequality to some realistic experiments. We hope that this inequality will be very useful in testing quantum work relations for many body systems and will open up further thoughts in the area of quantum work relations and fluctuation theorems. Also, this inequality may be applied to probe quantum nonequilibrium phenomena in glassy systems and many body systems.

Acknowledgements: AKP thank A. K. Rajagopal for useful discussions.

[1] C. Jarzynski, Phys. Rev. Lett. 78, 2690 (1997).

[2] G. E. Crooks, Phys. Rev. E 60, 2721 (1999).

[3] J. Kurchan, arXiv:cond-mat/0007360.

[4] T. Monnai, Phys. Rev. E 72, 027102 (2005).

[5] H. Tasaki, arXiv:cond-mat/0009244.

[6] D. Andrieux et al New J. of Phys. 11, 043014 (2009).

[7] T. Monnai, Phys. Rev. E 81, 011129 (2010).

[8] T. Monnai and S. Tasaki, arXiv: cond-mat/0308337.

[9] P. Talkner, E. Lutz, and P. Hänggi, Phys. Rev. E 75, 050102 (2007).

[10] S. Mukamel, Phys. Rev. Lett. 90, 170604 (2003).

[11] M. Campisi, P. Talkner, and P. Hänggi, Phys. Rev. Lett, 102, 210401 (2009).

[12] D. Andrieux and P. Gaspard, Phys. Rev. Lett. 1000, 230404 (2008).

[13] R. Kubo, J. Phys. Soc. Jpn. 12, 570 (1957).

[14] L. Onsager, Phys. Rev. 37, 405 (1931).

[15] R. P. Feynman, Statistical Mechanics, Frontiers in Physics, W. A. Benjamin (1972). 